\title{
Research on the Applications of Multimedia Technology under the Current Epoch of Internet Plus
}

\author{
Shenghong Liu \\ Hankou University, \\ Wuhan 430212 Hubei,P.R.China
}

\begin{abstract}
In this paper, we conduct research on the applications of the multimedia technology under the current epoch of Internet plus. Advances in science and technology to drive the move forward with the development of information industry, the development of computer network technology has been largely and the enhancement, it also promotes the artistic expression of visual communication design continuously toward the direction of diversification, enrichment, and for designers in terms of visual communication design provides more adequate performance of the carrier and multimedia technology as the medium of the spread of the modern art design essential way and, in the communication design teaching process in the role of the information transmission and communication that is an important communication platform. Additionally, we integrate the background of Internet plus to form modified theoretical analysis to predict the future of the multimedia technology.
\end{abstract}

Keywords- Multimedia Technology, Applications. Internet Plus, Current Epoch, Literature Review.

\section{Introduction}

Multimedia technology is the modern social information technology industry rapid development, as widely used, one of the means of active, competing with each other for new electronic technology and continuous development of the core and focus. Multimedia technology comprehensive application of rich information, images, sound and text data based on the imitation of human brain thinking, logical processing skills, using people's habits, the closest natural pattern to accept and process information, significantly improve the computer's ability to process information.
Multimedia technology is a technology and with printing technology, computer technology, radio broadcasting television after the new technology revolution, this article expounds the connotation of computer multimedia technology, application, characteristics and application situation, analyzes its development trend and way to expand, to create efficient and quick information network, expand the computer multimedia technology application service way, to realize the entire development of high quality that has a positive and effective role in promoting [1-3].

Generally the applications of the multimedia technology could be summarized as the listed aspects. (1) Multimedia function in modern network communication, the network communication technology development made the pace of history. Videophone, video conference, e-mail and so on application of the technology is the development and application of multimedia technology as also will continue to develop in wider areas to change the life of human beings. (2) Multimedia is born from the beginning, its commercial value is paid great attention by people, whether it is the birth of a single media silent TV or core Internet applications today that are potentially huge commercial value. (3) Multimedia technology applied in the field of education, changed the traditional way of teaching to promote the development of the education. In the multimedia teaching environment, the learner's thinking more active, participation becomes higher and the learning efficiency was improved, so as to promote the development of the whole education. (4) Electronic publishing. Traditional 
publications capacity and large volume, high cost, bad for carrying and retrieval and based on multimedia technology electronic products, it can not only for storage of the word, but also to the information such as voice and image can be easily stored, and in terms of capacity, volume and cost has a great advantage [4].

The formation of the Internet is built on the basis of computer hardware for its development should begin from transmission and exchange of information. The simple principle of communication is to be able to source information, pass through a medium, and then achieve the receiving end of receiving information. But the existence of the Internet, and is closely related to communication. The Internet is the carrier of it through the network, information transfer between. From the analysis on the meaning of the most simple, one of the Internet application in place of information transfer. Therefore, this article will be detailed analysis from various angles, to explore the comprehensive computer Internet in the current application. We firstly show the application scenarios of the multimedia as follows.

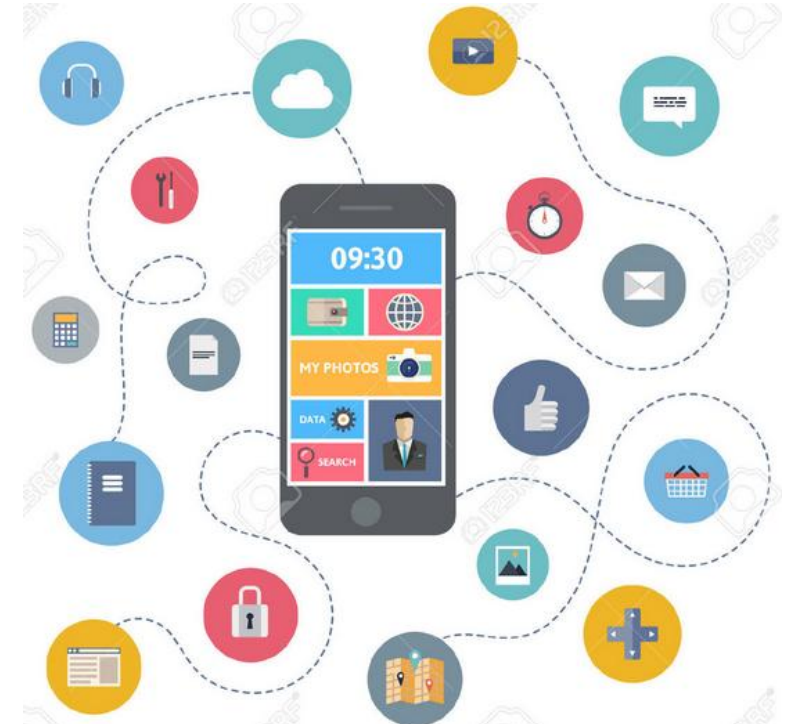

Figure 1. The Primary Application Scenarios of the Modern Multimedia Technology

In this paper, we conduct research on the applications of multimedia technology under the current epoch of Internet plus. Human civilization from agricultural development to the industrial age, until now the information age as is mainly of productivity. Historically, information transmission has been played a very important role. The current information age, the main is the information communication and transmission. The Internet became the carrier of information, as the angel of the message. At the same time, the Internet to take the functions of the information storage, people put a lot of information on the Internet that become a kind of information broadcasting.

\section{The Proposed Methodology}

The Primary Components of the Multimedia System. Multimedia computer technology mainly for the processing of objects including 3D graphics and surround stereo audio and full-color full screen image, etc. due to the problems faced by the digital computer including data, audio, graphics, text, language, image and video and other media more comprehensive problems, the load by the analog conversion throughput, storage and transmission of digital information. Data compression technology refers to the graphics, audio and video signal compression, storage and the distribution, we through improving the efficiency of the communication mains transmission can provide more effective means for the technology application, at the same time, computer control center can be real-time monitoring and processing of audio and video information, which can guarantee the output of high quality sound, practical video program. As known to all, multimedia computer technology popularization gradually, it can release a large number of multimedia information resources on the web, whether or not some information resources can be effective organization management and retrieval will rely on the index of the image and media content [5-6].

The application of multimedia technology and development to some extent, also contributed to the computer is more perfect to support multimedia cooperative work environment, 
interactive and the dynamic multimedia technology can be created under the condition of broadband is more vivid and lifelike three-dimensional scene, users can use multimedia video equipment and entertainment tool set office equipment in the multimedia terminal controller, in any corner of the world, we can achieve with thousands of miles away, colleagues, friends and family for video calls and the excellent image quality. To meet the requirements of multimedia network, we still need to do some in-depth research and development, the relevant software to enhance the multimedia computer control terminal has the better parts and high intelligence, multimedia computer identification and control terminal can add text information input, Chinese information identification and input, natural language cognitive and machine translation, graphic information identification and intelligent recognition, robot vision and computer vision, etc. The figure two shows the multimedia focusing points in China and the US.

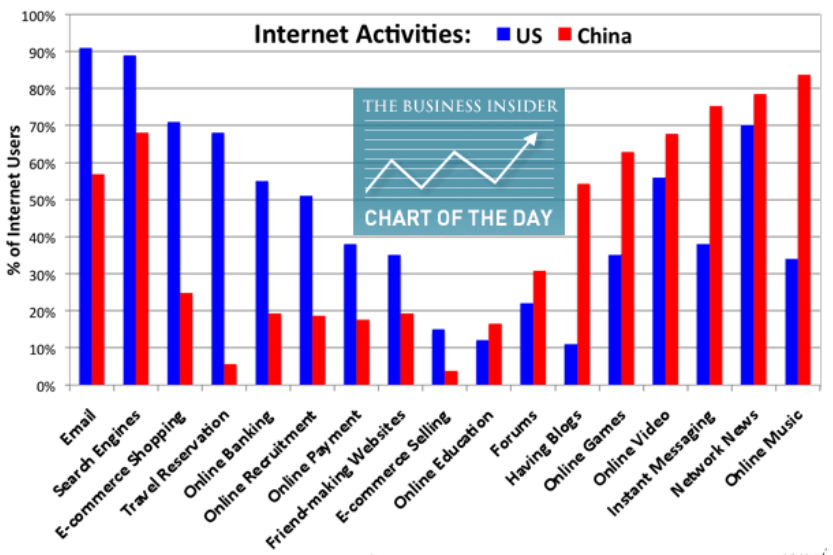

Figure 2. The Internet Multimedia Focusing

Points in China and the US

The Features of Internet Plus. With the development of the Internet industry, the penetration and speed of innovation in the field of consumption gradually from the explosive development of the original to the rapid growth of the more stable while the real economy as a whole is still in a state of the informationization as the extensive development of insufficient, the penetration of the Internet development informationization provides new impetus and platforms for enterprise. Industrialization and informatization process of China starts relatively late in the developed countries, and the lag of the Internet is not obvious and especially in the field of mobile Internet development is relatively advanced. Build critical resources industry development platform, set power to do relevant industry bigger and stronger, in the industrialization and informatization degree after reaching a certain level crossing into the industrial age of the Internet is an important mission to the China's Internet industry development. The figure three shows the developmental trend of Internet in China [7]. 
Number of Chinese Internet Users

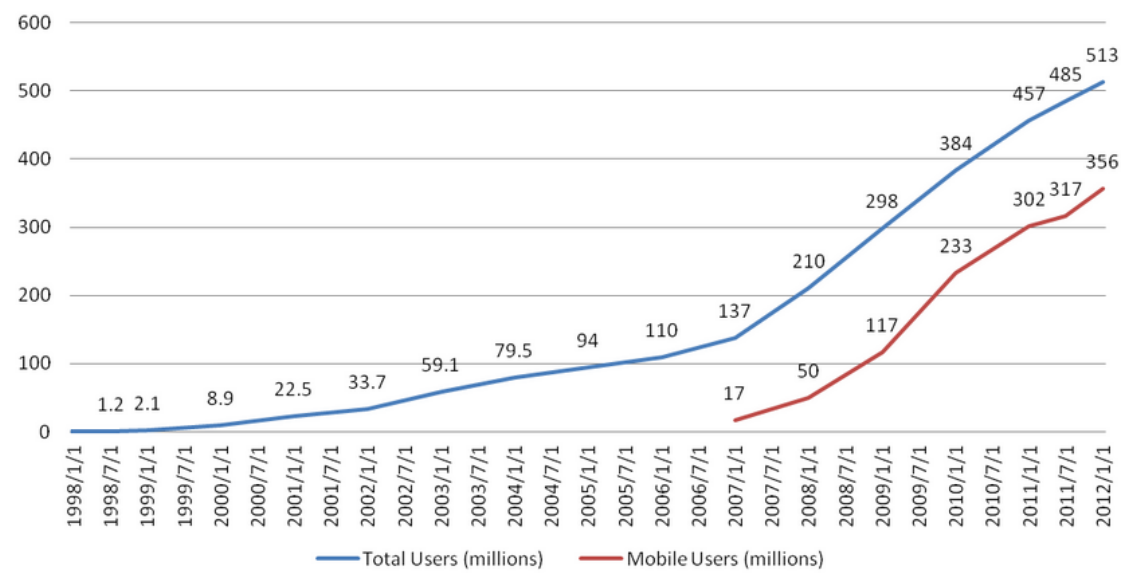

Figure 3. The Developmental Trend of Internet in China

The Internet become the global flow of information domain, is sure to be not only puts forward new challenges to the new international order, and brought plenty of opportunities for new movement. New order must consider the diversity of the subject and value, on the one hand, rely on developing countries and the emerging countries, on the other hand to the transcend national state, integrating supra-national institutions, non-governmental organizations, the power of the grassroots civil society, looking for ideas, reshape subject, the reconstruction system, to search for the Internet environment in the possibility of a new world information and communication order.

The Core Features of the Multimedia Technology. In multimedia platform, a variety of media, how to organize a variety of media in the space, the interaction between the media, how to describe and explain how to arrange on time is multimedia in the expression information must consider the question, the role and influence of their interaction is an indispensable component in the performance. We summarize the features of the multimedia techniques in the listed sections [8].

Interaction is an important characteristic of multimedia technology present use of computer multimedia technology as users can carry on the many kinds of media information interaction with a computer.
Banks, for example, the touch-screen computers installed in the main hall is able to effectively achieve the customer with the switchboard system and interactive, thereby saving a lot of artificial mechanical services, reduce the management cost, to meet customer demand more of the services.

Diversity is mainly for computer, mainly manifested in the diversity of information carrier as it can also be referred to as the multidimensional nature of the media. Computer processing range and variety of information media has not only confined to voice and image.

Integration is mainly refers to: the use of multimedia technology will be organically integrated, multimedia information to text voice and the image fusion. Then the comprehensive complete multimedia information is passed to the clients.

The Trend and Applications of Multimedia. The future study of multimedia, basically have the following research areas: data compression, multimedia information characteristics and the modeling, multimedia information's organization and the management, multimedia information expression and interaction communication and distribution processing, multimedia software and hardware platform, virtual reality technology, 
multimedia application development. Looking to the future that blends of the interactive multimedia network and computer technology will become a multimedia development direction in the 21st century [9].

Interactive multimedia refers to not only can accept information from Internet, select information, also can send information, the information in the form of the multimedia transmission. Using this technology, people can shop at home, on demand of favorite TV program. Multimedia, as we have shown new technology is becoming a portable personal multimedia. The further potential applications can be reflected from the listed aspects. (1) The introduction and application of multimedia network teaching system. Multimedia network classroom software system that is also known as networked multimedia classroom software system, usually refers to the average or ordinary teaching network, on the basis of single room through the necessary software and hardware machine to connect the machine auxiliary equipment to realize teachers and students, achieve the real time interaction between each computer screen, voice switching, and have a variety of auxiliary teaching software system which has the function of the teaching management. (2) Reform in the field of the multimedia more journals. Recently, by means of multimedia editing academic journals of science and technology for processing and administrative management and issued publicity, has become a significant trend. The application of multimedia technology will lead to a comprehensive change of traditional editing means.

\section{Conclusion}

In this paper, we conduct research on the applications of the multimedia technology under the current epoch of Internet plus. In the field of information technology, multimedia technology as the most rapid development at the same time the most active content, for the sustainable development of the electronic technology in the new period and the core and basic focus of the competition. Multimedia technology integrated with computer technology, audio processing technology, the text information processing technology, image processing, animation, video technology and network communication technology with the expansion rapidly through information network and complete computer across regional and even global networking that can realize information resources sharing. Our research uses the Internet plus background to propose the novel perspective of enhancing the current and future developmental trend of multimedia technique which holds vital significance.

\section{Acknowledgement}

This paper is financially supported by the project: Research on the nixed classroom teaching mode for the course of fundamentals of college computer.

\section{References}

[1] Jun, Hou, and Chen Wen. "Experience on the Design of Multimedia Technology Experiments in Electronics Engineering and Communication Major." The Science Education Article Collects 1 (2014): 053.

[2] Hermes, Mary, and Kendall A. King. "Ojibwe language revitalization, multimedia technology, and family language learning." (2013).

[3] Richards, Reshan. "Screencasting: Exploring a Middle School Math Teacher's Beliefs and Practices Through the Use of Multimedia Technology." International Journal of Instructional Media 39.1 (2012).

[4] Ma, Yingying. "Research on Effectiveness of Digital Multimedia Technology to the Improving of Artistic Design Teaching." 2014 2nd International Conference on 
Education Technology and Information System (ICETIS 2014). Atlantis Press, 2014.

[5] Jones, Gareth JF. "An introduction to crowdsourcing for language and multimedia technology research." Information Retrieval Meets Information Visualization. Springer Berlin Heidelberg, 2013. 132-154.

[6] Ali, Asma Md, and Joan Richardson. "Web interactive multimedia technology: implementation from two perspectives." International Journal of New Computer Architectures and their Applications (IJNCAA) 2.1 (2012): 154-166.
[7] Gnagnarella, Patrizia, et al. "Nutritional Online Information for Cancer Patients: a Randomized Trial of an Internet Communication Plus Social Media Intervention." Journal of Cancer Education (2015): 1-9.

[8] Jianfeng, H. U. "Construction of Internet plus College Based on the Ecological Circle of Industry and Education." (2015).

[9] Zhou, Xingiian, and Shaonan Huang. "Value Chain Analysis and Reconstruction of Internet plus Logistics." (2015). 